\title{
L'ontologie variable des actants : pour une épistémologie renouvelée dans les recherches sur les réseaux
}

Mylène Hardy et Serge Agostinelli

\section{(2) OpenEdition Journals}

Édition électronique

URL : http://journals.openedition.org/communicationorganisation/4101

DOI : 10.4000/communicationorganisation. 4101

ISSN : 1775-3546

Éditeur

Presses universitaires de Bordeaux

\section{Édition imprimée}

Date de publication : 1 juin 2013

Pagination : 25-36

ISBN : 978-2-86781-883-7

ISSN : 1168-5549

Référence électronique

Mylène Hardy et Serge Agostinelli, «L'ontologie variable des actants : pour une épistémologie renouvelée dans les recherches sur les réseaux », Communication et organisation [En ligne], 43 | 2013, mis en ligne le 01 juin 2013, consulté le 04 mai 2019. URL : http://journals.openedition.org/ communicationorganisation/4101; DOI : 10.4000/communicationorganisation.4101 


\title{
L'ontologie variable des actants : pour une épistémologie renouvelée dans les recherches sur les réseaux
}

\author{
Mụlène Hordụ', Serge Agostinelli²
}

Les réseaux sociaux peuvent être envisagés sous l'angle sociotechnique du modélisateur et relient des utilisateurs entre eux; ils sont aussi artefactuels pour l'utilisateur et associent actants matériels et symboliques. Ainsi, les réseaux sociaux dépassent une structure composée d'utilisateurs. Ils constituent des formes organisationnelles complexes qui sont ontologiquement hétérogènes. L'ontologie des actants et leur variété se positionnent aujourd'hui comme une difficulté de la modélisation des réseaux sociaux numériques.

\section{Ontologie des actants dans les approches en STIC et SHS}

Une ontologie est un système conceptuel destiné à fournir les notions élémentaires nécessaires à la formulation des connaissances dont on dispose sur un sujet donné (Bachimont, 2004). Un actant peut être une personne, un objet, une organisation qui contribue à un équilibre (Latour, 2005) ou un système en acte (Passeron, 2001). À partir de ces deux notions, les réseaux peuvent soit être envisagés par la prise en compte de la complexité des interactions entre de multiples agents ; soit par leur hétérogénéité ou suivant leurs actants hétérogènes.

\section{Homogénéité ontologique dans les approches formelles}

Les approches formelles analysent et modélisent les réseaux sous l'angle des actants homogènes. Par exemple, la description des réseaux complexes peut passer par une méthode analytique permettant de calculer les corrélations entre les degrés de sites web (Barrat, 2011).

1 Mylène Hardy est Docteur en Sciences de l'Information et de la Communication, enseignant-chercheur dans plusieurs universités chinoises. Ses recherches portent sur les approches réticulaires en communication des organisations, notamment dans le cadre des activités d'intelligence économique en Chine ; hardymylene@ yahoo.com

2 LSIS, UMR CNRS 7296, Professeur à l’Université Aix-Marseille ; serge.agostinelli@univ-amu.fr 
Ce type d'analyse peut s'appliquer à tous types de réseaux et on peut y voir une nouvelle Science capable d'expliquer de nombreux domaines du monde (Barabasi, 2002). Elle cherche à prendre en compte la complexité des interactions entre de multiples agents afin de faire émerger les propriétés des catégories d'interactions. Cette approche place les réseaux dans la catégorie des systèmes complexes et auto-organisés. Elle reste toutefois (post) positiviste et se fonde sur un réductionnisme ou un déterminisme (Morçöl, 2001) qui limite l'acteur à un même type d'action : la réduction porte sur la nature des actants et sur celle de leurs interactions car les calculs statistiques ne peuvent être effectués que dans des réseaux possédant des actants de même nature et un type d'interaction. La non-linéarité des réseaux est produite de manière déterministe par la complexité des relations.

Cette approche formelle est largement reprise pour l'analyse des réseaux sociaux (Hanneman et Riddle, 2005 ; Garton, Haythornthwaite et Wellman, 1997) sous une forme structurale qui considère un nombre de relations restreintes entre les nœuds du réseau (Scott, 2000). Elle appréhende les réseaux de manière statique et réduit fortement l'hétérogénéité de l'organisation (Monge et Contractor, 2001) avec des résultats globalement classiques (Eve, 2002). Elle réaffirme la primauté de la configuration, de nouvelles propriétés macro émergeant à partir de règles d'interactions locales (Goldstein, 1999). C'est l'organisation dans sa dualité structurelle comme produit d'interactions et contraintes sur ces interactions qui confère une position originale à ces recherches (Degenne et Forsé, 2004).

\section{Hétérogénéité ontologique de la théorie de l'acteur-réseau}

À l'opposé des approches formelles sur les réseaux physiques et sociaux, d'autres approches ont la capacité d'appréhender les réseaux hétérogènes d'actants et même les réseaux d'actants hétérogènes (Callon, 1986). Avec une approche anthropologique de l'urbanisation, l'école de Manchester fonde explicitement le concept de réseau pour décrire la texture des liens (Gribaudi, 1998), la multiplexité des relations et l'hétérogénéité de l'identité des acteurs (Eve, 2002). Avec une approche constructiviste et situationniste, la théorie de l'acteur-réseau $(\mathrm{ANT})^{3}$ cherche à rapprocher le micro et le macro (Callon, 2006). Elle donne une place primordiale et agissante aux non-humains. Elle ne parle plus d'acteurs, mais d'actants, et, même si la théorie de l'acteur-réseau a été critiquée pour son transfert anthropologique trop fort sur les non humains (Collins \& Yearley, 1992), l'originalité de son apport reste incontestable (Brechet \& Desreumaux, 2007). Dans la théorie de l'acteur-réseau en effet, non seulement les actants peuvent avoir des ontologies diverses, mais sont eux-mêmes des réseaux, l'ensemble formant des collectifs hybrides (Callon et Latour, 2006). L'action est délocalisée et chaque

3 Callon (2006) préfère traduire «network-actor theory » par «sociologie de l'acteur-réseau », mais nous avons préféré garder une traduction française au plus proche de l'anglais. 
actant peut ainsi télé-agir et, en tant que médiateur, traduire, représenter les formes et discours de l'organisation (Cooren, 2006, 2000). Ces actants sont des artefacts communicationnels (Agostinelli, 2003) qui associent en un moment et espace donnés, une hétérogénéité sociale situationnelle (Latour, 2005). La mobilisation d'un actant entraîne alors tous les autres, actants collectifs, qui lui sont rattachés. Il ne nous semble cependant pas possible de donner aux non-humains une capacité d'action égale à celle des humains. Les acteurs humains possèdent une caractéristique différente des autres actants, la capacité de modifier les règles d'action, et donc d'engendrer une reconfiguration des autres réseaux. La théorie de la structuration stipule que la reproduction-modification de la structure sociale s'effectue notamment à travers les conséquences non intentionnelles de l'action (Giddens, 2005). Pour les acteurs humains, l'action peut être intentionnelle comme processus en cours et comme objet de la connaissance comme future action anticipée (Schütz, 1987). Dès lors, si on peut supposer les non humains peuvent avoir des conséquences non intentionnelles qui permettront l'évolution de la structure, seule l'action intentionnelle humaine est capable de modifier la structure de manière intentionnelle tout en la reproduisant. L'action non intentionnelle n'est qu'une réponse réactive (Giddens, 2005) et non pas une action. En tous les cas, si l'on décide que les objets produisent des actions et que l'on considère qu'un objet produit une action intentionnelle, c'est que cette intention n'est que la traduction des acteurs humains qui ont produit cet objet.

Les réseaux organisés humains ont des caractéristiques culturelles que ne possèdent pas les autres actants. La question du sens pose problème dans l'analyse des réseaux car il ne peut être attribué que par des actants humains (Hardy et Agostinelli, 2008). Une telle analyse conduit à penser qu'un modèle de réseaux d'actants à ontologie variable devra nécessairement :

- donner aux actants humains une capacité d'action supérieure à celle des autres actants, en leur attribuant par exemple comme le fait Carley la capacité de modifier le réseau ;

- utiliser pour ce faire un double réseau, celui des actants physiques, et celui des actants conceptuels.

Ce modèle pourrait par exemple utiliser le modèle de la coorientation proposé par Taylor (2006) qui relient deux mondes, socio-matériel et conceptuel, le monde conceptuel correspondant dans notre modèle au réseau cognitif qui constitue le système de règles de la structure sociale, le monde socio-matériel en composant les ressources. Les actants non humains n'ont pas la capacité de modifier les systèmes de règles, alors que les actants humains les modifient continuellement. À travers leurs interactions communicatives, les acteurs donnent en effet du sens à leurs actions, et le recadrage effectué à travers ces interactions modifie les règles constitutives et normatives de l'interaction. Ces 
cadres de l'interaction sont en fait eux-mêmes des méta-matrices constituées de réseaux sociocognitifs. Les liens entre les membres d'une même culture et le réseau socio-cognitif qui constitue cette culture seront plus denses que les liens qu'ils entretiendront avec d'autres cadres conceptuels et régulatoires. Un acteur pourra être membre de plusieurs cultures et à plusieurs niveaux de structuration différents. L'appartenance à une culture est donc au centre de l'organisation du réseau, puisque l'organisation ne peut émerger que si les acteurs partagent l'interprétation des règles qu'ils appliquent (Tsoukas, 2000).

\section{Hétérogénéité ontologique dans des réseaux d'interaction}

\section{Nécessité théorique des réseaux hiérarchisés}

Penser les réseaux comme hiérarchisés peut être un moyen de résoudre la question ontologique des actants à la fois en STIC et en SHS. Notre analyse envisage la complexité sociale à travers une multiplicité de réseaux dynamiques multi-niveaux permettant de prendre en compte l'ontologie variable des actants. Les actants sont à la fois des unités d'ontologie variable et des acteurs-réseaux d'action liés à des réseaux de règles d'action (Hardy \& Agostinelli, 2008).

Relier les approches technocentrées des STIC avec les approches anthropocentrées et non hiérarchique des SHS semble, à première vue, difficile mais non impossible si on pose l'émergence comme propriété des réseaux et si l'on apprécie l'importance d'un actant à partir du nombre de connections réticulaires qui le lient avec d'autres actants (Latour, 1996). Les objets du réseau sont alors définis par la stabilité des liens avec d'autres entités et une grammaire ou une syntaxe stable de ces liens (Law 2002,2000). Les objets ainsi pensés ont une autre caractéristique, celle d'une « continuité fluide », qui fait que les objets ne sont pas fixes mais ont des limites mouvantes tout en gardant une certaine stabilité. C'est d'ailleurs cet aspect plat qui permet dans l'ANT aux actants d'avoir eux-mêmes une ontologie réticulaire. Pourtant, cet aspect plat du réseau ne semble pas permettre l'émergence de propriétés nouvelles comme permettent de l'envisager les réseaux structurés. Il nous semble donc difficile d'envisager la stabilité des liens en dehors d'une émergence de forme structurée. La construction d'entités macro, de Léviathans institutionnels, ne semble possible que s'il y a structuration et donc, cristallisation physique et symboliques d'entités de niveau supérieur. La traduction et le passage des discours cristallisés à travers un actant ne sont pas incompatible avec la structuration du réseau, à condition de supposer que la variabilité ontologique des actants permette à des actants de niveau de structuration différents d'être reliés ensemble.

En réalité, ce sont les aspects longitudinaux et la question du multi-niveaux qui intéressent l'ontologie variable des actants. L'analyse formelle des réseaux n'est pas incompatible avec un tel modèle (cf. : Snijders, 2011 ; Jourda et al., 
2007 ; Carley, 2003 ; Snijders et al., 1995). Toutefois, elle est encore limitée par le nombre de variables pouvant être pris en compte et par la nécessité de travailler sur des réseaux de taille relativement homogène ou de les fermer artificiellement. Ces limites font d'ailleurs l'objet de recherches (Snijders et Baerveldt, 2003) qui examinent la réunion de multiples réseaux. Bien que difficile à mettre en oeuvre et réduite à quelques niveaux, l'analyse multi-niveaux et surtout le concept de méta matrice permettent d'introduire dans l'analyse réticulaire l'hétérogénéité ontologique et donc une plus grande richesse socio-artefactuelle. Par exemple, l'étude de la configuration de réseaux terroristes (Carley, 2003) demande un modèle dans lequel dix réseaux sont interelliés. Ce modèle montre les connections entre des personnes, des connaissances/ressources, des événements/tâches, et des organisations. On croise ici le réseau des personnes avec celui des organisations et celui des événements afin d'obtenir une matrice d'appartenance des personnes aux organisations qui indique quelle personne a participé à tel événement. Les relations dans un réseau étant liées aux relations dans d'autres réseaux, le modèle permet d'observer la co-évolution des réseaux et les répercussions des changements effectués dans un réseau sur les autres. La non-linéarité des actions est d'autant plus forte que le modèle multiplie les types de liens entre les actants. Il prend donc en compte la variabilité ontologique de ces liens au même titre que celle des actants. Les acteurs sont réellement capables d'agir, et donc de modifier le réseau en fonction de la perception qu'ils en ont. La structuration dynamique de ces réseaux fait émerger de nouvelles propriétés qui n'existaient pas dans les réseaux séparés.

\section{Actants réticulaires et variabilité ontologique}

L'ANT n'envisage pas seulement des actants possédant chacun une nature différente, elle pense qu'un actant peut regrouper, en tant que réseau de voix traduites et cristallisées en lui, des natures différentes en une seule entité. C'est pourquoi nous parlons de variabilité qui apparaît à un moment donné dans le processus évolutif des réseaux. Les réseaux comportent un nombre infini de variables (changement d'aspect, de degré ou de valeur) et les STIC répondrent à cette variabilité car elles permettent la télé-présence des actants à travers les voix traduites en d'autres actants. Cependant, dans des réseaux hiérarchiques, on ne peut envisager la variabilité que comme le résultat de l'empilement de réseaux. Dans les réseaux aériens, un graphe avec plusieurs niveaux hiérarchiques selon le niveau d'interconnection des villes (Amiel et al., 2005) fait apparaître des profondeurs différenciées. Les actants de niveaux de structuration différents sont reliés ensemble, et on peut explorer les réseaux qui composent certains des actants à des niveaux inférieurs. Pour reprendre l'exemple de Callon (2006), l'action collective d'un groupe d'actants, une entreprise automobile pourrait se cristalliser à un niveau supérieur par un actant traduisant cette action, une voiture. Mais même dans une conception 
réticulaire hiérarchisée, cet actant de niveau supérieur pourrait tout à fait être mis en relation avec des actants individus de niveau inférieur, permettant la circulation des voix entre les niveaux.

\section{La question de l'espace au carrefour d'un renouveau théorique}

Un autre moyen de croiser les STIC et les SHS serait de travailler à partir de la notion d'espace, en travaillant de manière interdisciplinaire. À l'exemple du «tournant linguistique » qui a affecté les méthodes et les concepts en vigueur dans les sciences sociales, l'idée du « tournant spatial » s'est récemment imposée comme un nouveau dispositif épistémique (Besse 2010, p. 193).

\section{Distance et échelle : les catégories de l'espace à la croisée des réflexions STIC et SHS}

Penser le monde que sont les réseaux implique un changement topologique. "Plutôt que de penser en termes de surfaces - deux dimensions - ou de sphères - trois dimensions - l'on nous demande de penser en termes de nodes qui ont autant de dimensions qu'ils ont de connections. (...) La force ne vient pas de la concentration, de la pureté et de l'unité, mais de la dissémination, de l'hérogénéité et du tressage minutieux des liens faibles » (Latour, 1996, p. 3) ${ }^{4}$. Nous avons vu que, dans sa réflexion ontologique sur les objets, Law (2002, 2000) montrait que les objets, qui sont des " espaces réticulaires ", ont une forme stable tant qu'ils gardent une syntaxe stable des relations qui unissent les éléments les composant. Les objets-réseaux ne sont pas placés dans un espace donné, mais performent leur espace lorsqu'ils s'énactent. Selon Law (2000), les objets sont « des stabilités qui jaillissent de performances d'invariances formelles dans plus d'un topo »(p. 9). La multiplicité topologique est réticulaire. Pour penser les objets-réseaux, l'on ne peut penser l'espace qui les « comprend " que comme réseau lui-même. C'est cette idée que Maintz $(2009,2008)$ met en ouvre pour examiner le mélange et la continuité entre les espaces de communication physique en face à face et ceux médiatisés par l'informatique. Dès lors, les outils mathématiques utilisés par les STIC permettent de donner aux catégories de proximité et de distance une nouvelle définition, en termes de connexions, ce qui permet de prendre en compte l'espace créé par les actants eux-mêmes. Contrairement à Latour (1996), il nous semble qu'il ne faut pas se défaire de cadres de pensée existants comme ceux d'échelle ou de distance, mais changer leur conception, en s'appuyant sur les disciplines qui les étudient plus particulièrement, comme la géographie. Le réseau apporte un éclairage nouveau à la notion d'échelle : il ne s'agit plus de définir a priori ce qui est micro ou macro, mais de les définir en situation. «Une échelle n'est pas un niveau ou une grandeur pré-définie ou discrète dans une hiérarchie d'unités spatiales de taille de plus en plus petite ou de plus en plus

4 Traduction personnelle. 
grande. Une échelle est plutôt toujours construite et contestée socialement ; son sens spécifique découle des contextes dans lesquels le langage de l'échelle est déployé par diverses organisations sociales, politiques et économiques. Une échelle peut être hiérarchique, peut être réticulaire et elle peut être aussi les deux " (Jonas, 2011, p. 4015). Les réseaux sociaux nous semblent un bon exemple de ce que peut être une échelle à la fois réticulaire et hiérarchique. Les dernières avancées des STIC se rapprochent en ce sens de plus en plus des SHS. Par exemple, Li et Lin (2010) cherchent à extraire automatiquement de l'information dans des réseaux sociaux hétérogènes comportant plusieurs types de nodes interreliés par plusieurs types de liens et proposent d'examiner le problème à partir d'un point de vue egocentrique, c'est-à-dire à partir d'un node particulier. Ainsi, le point de vue sur le réseau devient celui du node, ce qui permet à l'utilisateur de trouver l'information liée au point particulier qui l'intéresse. Il s'agit là, comme le disent les auteurs, d'un point de vue micro ; mais l'on voit bien ici que l'échelle, loin d'être fixe, est flexible, puisque le point de vue peut se déplacer dans le réseau selon les besoins de l'utilisateur et que l'analyse proposée pourra indiquer les informations à des distances diverses (informations plus ou moins centrales par rapport au point de départ). Ici, les notions d'espace, d'échelle et de distance sont bien les effets du réseau, pour reprendre l'ANT, et non pas des catégories prexistantes. Elles sont construites par les actants et s'auto-organisent en situation.

\section{Penser les concepts comme dynamiques et l'ontologie comme latente}

Dans l'ANT, le réseau devient une métaphore qui met en avant le local (la situation spatio-temporelle) et la flexibilité face à l'universel et la dureté. Plutôt que d'opposer les points de vue STIC et SHS à partir de positions fixes dans le temps, nous préférons les relier en pensant les concepts à partir des liens dynamiques qui les lient. Le concept de variabilité ontologique ne peut pas s'imaginer si l'on n'accepte pas d'abord la variabilité conceptuelle; par exemple la possibilité de penser l'universelle localité ou la dureté flexible, ce qui peut se penser dans d'autres cultures épistémologiques. Dans la culture chinoise, par exemple, les opposés forment une seule entité qui se déforme et se reforme selon que l'un ou l'autre prend le pas sur l'autre, sans que ni l'un ni l'autre n'en disparaissent tout à fait. Cette conception d'une latence ontologique nous semble primordiale, car elle permet non seulement de penser le rapprochement plutôt que l'opposition entre STIC et SHS, mais surtout parce que, appliquée plus directement au problème de la variabilité des actants, elle permet de résoudre la dichotomie des points de vue STIC et SHS en attribuant à un actant d'un réseau social une ontologie propre (donc l'acceptation qu'un réseau puisse se structurer localement et en situation de manière hiérarchique pour faire émerger une ontologie particulière) tout en

5 Traduction personnelle. 
gardant une possibilité de variation. Cette latence pourrait s'exprimer en STIC par des outils mathématiques statistiques, comme ceux utilisés en logique floue, même si les outils mathématiques actuels sont encore extrêmement réductionnistes.

\section{Conclusion}

Nous avons fait le pari dans notre texte d'un dialogue possible entre les approches STIC et les approches SHS. Nous pensons en effet que malgré les différences, voire les oppositions entre ces approches, un certain rapprochement est possible, facilité notamment par l'évolution des recherches sur les réseaux sociaux, la complexification des outils mathématiques statistiques servant à décrire leur dynamique, ainsi que l'augmentation des capacités de calcul des machines sur lesquelles sont expérimentés les modèles, pour l'instant encore très réductionnistes, des réseaux sociotechniques qui constituent le monde matériel. La seule limite à un rapprochement, outre un défaut d'ouverture d'esprit, est une limite de calcul, les machines ne pouvant actuellement pas encore appréhender l'infini, cette notion se trouvant pourtant à la base des théories de nature plus anthropologique sur les réseaux sociaux. En effet, la caractéristique des systèmes auto-organisés comme peuvent l'être les réseaux sociotechniques est que le changement qui mène à l'organisation du réseau est dû à une impulsion interne et non pas externe. Or, dans les systèmes sociotechniques, c'est bien la capacité humaine à modifier les réseaux de règles sociocognitives qui crée cette perturbation interne permettant l'autoorganisation du réseau d'actants. En dehors du problème du réductionnisme engendré par les limitations en puissance de calcul, notre modèle comporte plusieurs points que la recherche future pourrait explorer :

la difficulté à penser à la fois les actants comme ontologiquement fixes dans certains cas (objets, humains) et variables dans d'autres cas (variabilité réticulaire des actants symboliques);

les actants symboliques posent problème, car les liens avec les autres actants (socio-matériels) peuvent être parfois plus complexes qu'un simple modèle avec deux réseaux co-orientés pourrait le laisser penser. En effet, ces actants symboliques sont nécessairement réticulaires et peuvent être à la fois des actants sur lesquels portent les interactions communicatives humaines, tout se trouvant à une échelle supérieure contraignant le réseau socio-matériel hiérarchiquement inférieur ;

la difficulté de penser la direction des liens dans certains cas : les réseaux de règles contraignent l'action humaine ou non humaine. Mais un actant symbolique n'a pas nécessairement une relation contraignante sur l'action, notamment sur l'action non humaine.

Il faudrait donc explorer plus avant les différents types de liens possibles entre les différents types d'actants. En effet, si la question de la variabilité ontologique des actants est centrale pour les futures études sur les réseaux 
organisationnels, celle de la variabilité des liens ne l'est pas moins, d'autant plus que les liens actionnels entre les actants sont eux-mêmes des réseaux sociocognitifs.

\section{BIBLIOGRAPHIE}

AGOSTINELLI S., Les nouveaux outils de communication des savoirs, Paris, L'Harmattan, 2003.

AMIEL M., MELANCON G., et ROZENBLAT C., " Réseaux multi-niveaux : l'exemple des échanges aériens mondiaux de passagers ", M@ppemonde, 79(3), 2005, http://mappemonde.mgm.fr/num7/articles/art05302.html

BACHIMONT B., Arts et sciences du numérique : Ingénierie des connaissances et critique de la raison computationnelle, Université de Technologie de Compiègne, 2004.

BARABASI A. L, Linked: The New Science of Networks, Cambridge, Perseus, 2002.

BARRAT A., "La notion de réseaux complexes ", Médias011, Y a-t-il une richesse des réseaux? Aix-en-Provence : Université Paul Cézanne, 2011, http://www.medias011. univ-cezanne.fr

BESSE J.-M., " De la géographie comme dimension de la culture », L'espace géographique, 39 (3), 2010, p. 193-196.

BRECHET J.-P., DESREUMAUX A., " Que faire de l'ANT en management stratégique ", AIMS, 2007, http://www.strategie-aims.com/events/conferences/6xviieme-conference-de-1-aims/communications/1702-que-faire-de-lant-enmanagement-strategique/download

CALLON M., "Some elements of a sociology of translation : domestication of the scallops and the fishermen of St Brieuc bay ", in John LAW ed., Power, Action and Belief: A New Sociology of Knowledge, London, Routledge, 1986, p. 196-233.

CALLON M., "Sociologie de l'acteur-réseau », in M. AKRICH, M. CALLON B., LATOUR eds., Sociologie de la traduction : textes fondateurs, Paris, Presses des Mines, 2006, p. 267-276.

CALLON M., LATOUR B., Le grand Léviathan s'apprivoise-t-il ? Sociologie de la traduction : textes fondateurs, Paris, Presses de Mines, 2006, p. 11-32.

CARLEY K., " Dynamic Network Analysis », in R. BREIGER, K. CARLEY et P. PATTISON eds., Dynamic Social Network Modeling and Analysis : Workshop Summary and Papers, Washington, National Academies Press, 2003, p. 132-145, http://www.nap. edu

COLLINS H., YEARLEY S., « Epistemological Chicken », in A. PICKERING ed., Science and Practice as Culture, Chicago, University of Chicago Press, 1992, p. 301-326.

COOREN F., The organizing property of communication, Amsterdam, John Benjamins Publishing Company, 2000.

COOREN F., "The organizational world as a plenum of agencies », in F. COOREN, J. TAYLOR et E. VAN EVERY eds., Communication as organizing : Empirical and 
theoretical approaches to the dynamic of text and conversation, Mahwah, Lawrence Erlbaum Associates, 2006, p. 81-100.

DEGENNE A., FORSÉ M., Les réseaux sociaux : Une approche structurale en sociologie, Paris, Armand Colin, 2004.

EVE M., « Deux traditions d'analyse des réseaux sociaux », Réseaux, 115(5), 2002, p. 183-212.

GARTON L. HAYTHORNTHWAITE C. et WELLMAN B., « Studying online social networks ", Journal of computer-mediated communication, 3(1), 1997, http://jcmc. indiana.edu/vol3/issue1/garton.html

GIDDENS A., La constitution de la société : Eléments d'une théorie de la structuration, Paris : PUF / Quadrige ( $1^{\circ}$ éd 1984), 2005.

GOLDSTEIN J., " Emergence as a Construct : History and Issues », Emergence, 1(1), 1999, p. 49-72.

GRIBAUDI M., Espaces, temporalités, stratifications : exercices sur les réseaux sociaux, Paris, EHESS, 1998.

HANNEMAN R., RIDDLE M., Introduction to social network methods, Riverside, University of California, 2005., http://faculty.ucr.edu/ hanneman/nettext/

HARDY M., AGOSTINELLI S., « Organization as a multi-dimensional network of actants mediated by an organizing network of cultural rules. What is an Organization ? " Materiality, Agency and Discourse, Montréal, Université de Montréal, 2008, http://archivesic.ccsd.cnrs.fr/ docs/00/48/90/99/PDF/ 09-Art2-MHSAComMontreal2008C1.pdf

JONAS A., "Scale and networks - Part I ", in J. AGNEW, J. DUNCAN eds, The Wiley-Blackwell companion to human geography, Chichester : Wiley-Blackwell, 2011, p. 387-403.

JOURDA M.-T., MOUNIER L., STOFER R., LAZEGA E., « Des poissons et des mares : l'analyse de réseaux multi-niveaux », Revue française de sociologie, 48(1), 2007, p. $93-131$.

LATOUR B., "On actor-network theory. A few clarifications plus more than a few complications ", Soziale welt, 47, 1996, p. 369-381, http:/www.bruno-latour.fr/sites/ default/files/P-67\%20ACTOR-NETWORK.pdf

LATOUR B., Reassembling the social : an introduction to Actor-Network-Theory, Oxford- New York, Oxford University Press, 2005.

LAW J., « Objects, spaces and Others », Working Paper, 2000, http://www.lancs.ac.uk/ fass/sociology/papers/law-objects-spaces-others.pdf

LAW J., "Objects and spaces ", Theory, Culture and Society, 19, 2002, p. 91-105.

LI C.-T., LIN S.-D., " Mining heterogeneous social networks for egocentric information abstraction ", in N. MEMON, R. ALHAJJ eds, From sociology to computing in social networks : theory, foundations and applications, Wien, Springer, 2010, p. 35-58.

MAINTZ J, Blended spaces : actor-network interactions of an internet-based e-learning course, Münster, LIT, 2009. 
MAINTZ J., "Synthesizing the face-to-face experience : e-learning practices and the constitution of place online ", Social geography, 3, 1-10, 2008, http://www.soc-geogr. net/3/1/2008/sg-3-1-2008.html

MONGE P., CONTRACTOR N., "Emergence of communication networks ", in F. JABLIN, L. PUTNAM eds., The new handbook of organizational communication, Advances in theory, research and methods, London, Sage, 2001, p. 441-502.

MORÇÖL G., "What is complexity science? postmodernist or postpositivist? », Emergence : Complexity et Organization, 3(1), 2001, p. 104-119.

PASSERON J.-C., "Acteur, agent, actant : personnages en quête d'un scénario introuvable ", Revue européenne des sciences sociales, 39(121), 2001, p. 15-30.

SCHÜTZ A., Le chercheur et le quotidien. Phénoménologie des sciences sociales, Paris, Méridiens Klincksieck, (1º́d. 1971), 1987.

SCOTT J., Social Networks Analysis : a handbook (2 $2^{\text {nd }}$ ed.), London, Sage, 2000.

SNIJDERS T., " Network dynamics », in J. SCOTT, P. CARRINGTON eds., The SAGE handbook of social network analysis, London, Sage, 2011, p. 501-534.

SNIJDERS T., BAERVELDT C., "A multilevel network study of the effect of delinquent behavior on friendship evolution ", Journal of mathematical sociology, (27), 2003, p. 123-151.

SNIJDERS T., SPREEN M. et ZWAGGSTRA R., «The use of multilevel modelling for analysing personal networks : Networks of cocaine users in an urban area ", Journal of quantitative anthropology, (5), 1995, p. 85-105.

TAYLOR J., "Coorientation : a conceptual framework », in F. COOREN, J. TAYLOR et E. VAN EVERY eds., Communication as organizing: Empirical and theoretical approaches to the dynamic of text and conversation, Mahwah, Lawrence Erlbaum Associates, 2006, p. 141-156.

TSOUKAS H., « Knowledge as action, organization as theory », Emergence, 2(4), 2000, p. 104-112.

Résumé : Cet article discute un modèle original qui permet de dépasser les antagonismes des différentes écoles de pensée qui s’intéressent à la modélisation des réseaux sociaux. Nous faisons ici le pari qu'un dialogue est possible entre les approches TIC et les approches SHS car les réseaux sont à la fois sociaux et techniques pour relier les utilisateurs entre eux. Les réseaux sont des formes d'organisations complexes ontologiquement hétérogènes.

Mots-clés : compatibilité, modèle, humanités, technologie.

Abstract : This paper presents an original model that can overcome the antagonisms of different schools of thought with regard to the modeling of social networks. We make here the bet that a dialogue is possible between the approaches ICTS and the human sciences because networks are at the same time social and technical to connect the users between them. Networks are forms of complex and heterogeneous organizations.

Keywords : Compatibility model, Humanities, Technology. 
